\title{
BMJ Open Dietary fatty acids in the secondary prevention of coronary heart disease: a systematic review, meta-analysis and meta-regression
}

\author{
Lukas Schwingshackl, Georg Hoffmann
} To cite: Schwingshackl L,
Hoffmann G. Dietary fatty acids in the secondary prevention of coronary heart disease: a systematic review, meta-analysis and metaregression. BMJ Open 2014;4:e004487. doi:10.1136/bmjopen-2013004487

- Prepublication history and additional material is available. To view please visit the journal (http://dx.doi.org/ 10.1136/bmjopen-2013004487).

Received 18 November 2013 Revised 31 March 2014 Accepted 1 April 2014

CrossMark

Faculty of Life Sciences, Department of Nutritional Sciences, University of Vienna, Vienna, Austria

Correspondence to Lukas Schwingshackl; lukas.schwingshackl@univie. ac.at

\begin{abstract}
Objective: Previous systematic reviews were not restricted to either primary or secondary prevention trials, this study aimed to investigate the effects of reduced and/or modified fat diets and dietary fatty acids on all-cause mortality, cardiovascular mortality and cardiovascular events in participants with established coronary heart disease.

Design: Systematic review, meta-analysis and univariate/multivariate meta-regression.
\end{abstract}

Eligibility and criteria for selecting studies:

Electronic searches for randomised controlled trials comparing reduced/modified fat diets versus control diets were performed in MEDLINE, EMBASE and the Cochrane Library.

Data extraction: Pooled effects were calculated using an inverse-variance random effect meta-analysis. Random effects univariate and multivariate metaregressions were performed including changes in all types of dietary fatty acids.

Results: Overall, 12 studies enrolling 7150 participants were included in the present systematic review. No significant risk reduction could be observed considering all-cause mortality (relative risk (RR) $0.92, p=0.60$; $\mathrm{I}^{2}=59 \%$ ) and cardiovascular mortality (RR $0.96, \mathrm{p}=0.84$; $\mathrm{I}^{2}=69 \%$ ), combined cardiovascular events (RR 0.85 , $\mathrm{p}=0.30 ; \mathrm{I}^{2}=75 \%$ ) and myocardial infarction (RR 0.76 , $p=0.13 ; l^{2}=55 \%$ ) comparing modified fat diets versus control diets. This results could be confirmed for the reduced fat versus control diets (RR $0.79, p=0.47$; $\left.I^{2}=0 \%\right),\left(\right.$ RR 0.93, $\left.p=0.66 ; l^{2}=0 \%\right),($ RR $0.93, p=0.71$; $\left.\mathrm{I}^{2}=57 \%\right)$ and (RR 1.18, $\left.p=0.26 ; \mathrm{I}^{2}=18 \%\right)$. The multivariate and univariate model showed no significant associations between the independent variables and the changes from saturated fat, monounsaturated fat, polyunsaturated fat and linoleic acid. Sensitivity analyses did not reveal a significant risk reduction for any outcome parameter when polyunsaturated fat was increased in exchange for saturated fat.

Conclusions: The present systematic review provides no evidence (moderate quality evidence) for the beneficial effects of reduced/modified fat diets in the secondary prevention of coronary heart disease. Recommending higher intakes of polyunsaturated fatty acids in replacement of saturated fatty acids was not associated with risk reduction.

\section{Strengths and limitations of this study}

- Twelve studies enrolling 7150 participants were included in the present meta-analysis and meta-regression.

- Replacing saturated fatty acids by polyunsaturated fatty acids showed no significant benefit in the secondary prevention of coronary heart disease.

- Some of the included studies date back to 50 years.

- Substantial heterogeneity was observed for several outcomes.

\section{INTRODUCTION}

Studies reporting an association between intake of dietary saturated fatty acids (SFA) and serum cholesterol levels go back to the 1950s and 1960s. ${ }^{12}$ Supported by the epidemiological data observing a correlation between SFA intake and coronary heart disease (CHD) mortality, these findings established a reduction of SFA consumption as a major focus of dietary recommendations in order to prevent the prevalence of $\mathrm{CHD}^{3}$ although Siri-Tarino et at observed no relationship between saturated fats and CHD, stroke and cardiovascular disease (CVD) following a meta-analysis including 21 prospective studies.

Exchanging SFA for polyunsaturated fatty acids (PUFA), monounsaturated fatty acids (MUFA), carbohydrates (CHO) or protein exerted different effects on blood lipids and lipoproteins. ${ }^{5}$ In a systematic review and meta-analysis of cohort studies and randomised controlled trials (RCTs), Skeaff and Miller $^{6}$ concluded that there is convincing evidence that replacement of SFA by PUFA decreases the risk of fatal CHD and CHD events; however they could not confirm the hypothesis of a direct association between SFA intake and CHD death. ${ }^{6}$ Furthermore, 
the authors inferred that replacing SFA with CHO had no relation to CHD. The follow-up final report from the FAO stated that SFA intake should not be higher than $10 \%$ of the total energy consumption and that SFA should be replaced with PUFA. ${ }^{7}$ In their meta-analysis of cohort studies, Jakobsen et al observed that replacing SFA with PUFA reduced the risk of coronary events by $13 \%$ and the risk of coronary deaths by $26 \%$, respectively. In contrast, replacement of SFA by CHO or MUFA marginally increased the risk of coronary events, whereas no significant effects on coronary death could be observed. Mozaffarian $e t$ a ${ }^{\ominus}$ investigated the effects of increasing PUFA in replacement of SFA, and observed a significant decrease in the risk of CHD or associated mortality rates, while Hooper $e t a l^{10}$ reported a reduction in cardiovascular risk subsequent to a long-term reduction or modification in dietary fat intake. In an update of their meta-analysis, the same research team suggested that lowering of SFA intake led to a $14 \%$ decrease of the risk of cardiovascular events, however without affecting the cardiovascular or total mortality rates. ${ }^{11}$ It should be noted that reduction in cardiovascular risk was not associated with total fat in this study, but rather with a modification in dietary fat without clarifying the ideal type of unsaturated fat to replace SFA. Nevertheless, the US Dietary Guidelines recommend that $<10 \%$ of total energy content (TEC) should come from SFA and that saturated fat should be replaced with MUFA and PUFA. ${ }^{12}$ In 2011, the American Heart Association (AHA) and the American College of Cardiology (ACC) published a joint guideline endorsing less than 7\% of TEC in the form of SFA for patients with coronary as well as other atherosclerotic vascular diseases. ${ }^{13}$ Since previous systematic reviews were not restricted to either primary or secondary prevention trials, this study aimed to investigate the effects of reduced/modified fat diets versus control diets on allcause mortality, cardiovascular mortality, cardiovascular events (myocardial infarction, stroke) in participants with established CHD. The aim of the meta-regression was to include clinical outcomes and all dietary fatty acid changes in a univariate and multivariate model.

\section{MATERIALS AND METHODS}

\section{Literature search}

Queries of literature were performed using the electronic databases MEDLINE, EMBASE and the Cochrane Trial Register (until February 2014, respectively) with restrictions to RCTs, but no restrictions to language and calendar date using the following search terms: (dietary fat OR fatty acids OR low fat diet OR modified fat diet) in combination with (secondary prevention OR cardiovascular disease OR myocardial infartion OR coronary heart disease). Moreover, the reference lists from retrieved articles, systematic reviews and meta-analyses were checked to search for further relevant studies. This systematic review was planned, conducted and reported in adherence to the standards of quality for reporting meta-analyses. ${ }^{14}$ Literature search was conducted independently by both the authors, with disagreements resolved by consensus.

\section{Eligibility criteria}

Studies were included in the meta-analysis if they met all of the following criteria: (1) randomised controlled design; (2) minimum intervention period with a follow-up of 12 months; (3) comparing a reduced fat $(<30 \%$ of TEC) and/or modified fat diet versus control diet (SFA, MUFA, PUFA, linolenic acid and $\alpha$-linolenic acid values were either extracted from intervention/ dietary protocols or calculated from published data); (4) assessment of the 'outcome of interest' markers: allcause mortality, cardiovascular mortality, combined cardiovascular events, myocardial infarctions (fatal and non-fatal); (5) report of the number of events and sample size for each group and (6) only participants with established CHD (survivor of myocardial infarction, stable/unstable angina pectoris, acute coronary insufficiency) or coronary artery disease (CAD, verified by coronary angiography).

\section{Types of intervention}

The focus of this systematic review was set on examining the effects of reduced/modified fat diets as compared with control diets on 'hard' clinical endpoints.

\section{Risk of bias assessment and quality assessment}

Full copies of studies were independently assessed for methodological quality by both the authors using the risk of bias assessment tool by the Cochrane Collaboration. The following sources of bias were detected: selection bias (random sequence generation, allocation concealment), performance/detection bias (blinding of participants and personnel, blinding of outcome assessment), attrition bias (incomplete data outcome), reporting bias (selective reporting) and other bias (figure 1). ${ }^{15} 16$

\section{Data extraction and statistical analysis}

The following data were extracted from each study: the first author's last name, publication year, study duration, participant's sex and age, body mass index, sample size, SFA, MUFA, PUFA, linoleic acid, $\alpha$-linolenic acid, dietary cholesterol content of intervention protocol or dietary protocol, caloric intake, information on supplements, primary outcomes, number of events. For each outcome measure of interest, a random-effects inverse-variance meta-analysis was performed in order to determine the pooled effect of the intervention in terms of relative risks (RRs) and number of events of the reduced fat versus control diets, and modified fat versus control groups. All data were analysed using the REVIEW MANAGER V.5.1 software, provided by the Cochrane Collaboration (http://ims.cochrane.org/revman). Heterogeneity between trial results was tested with a standard $\chi^{2}$ test. The $\mathrm{I}^{2}$ parameter was used to quantify 


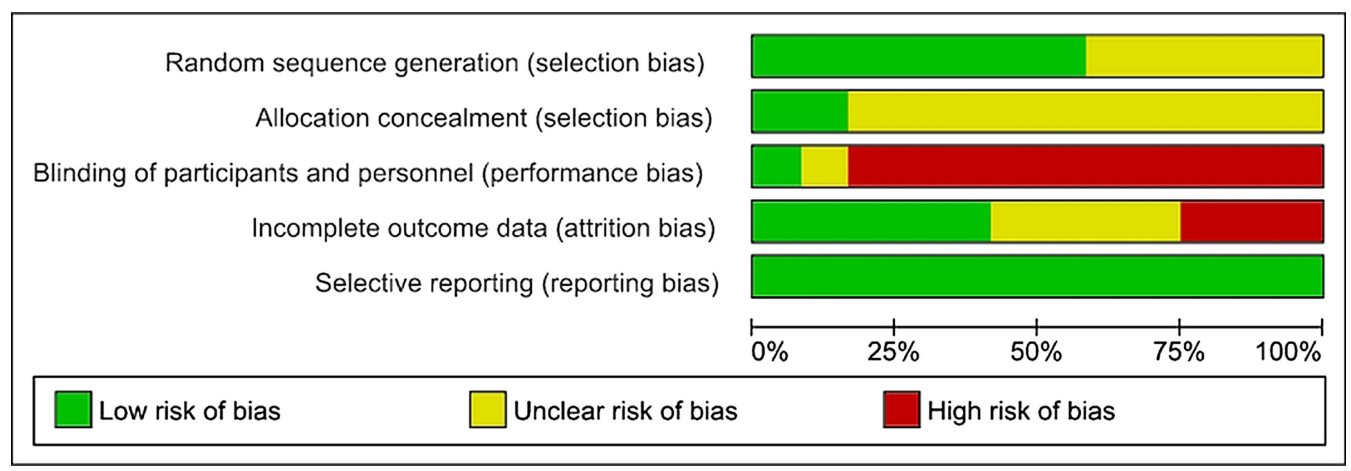

Figure 1 Risk of bias assessment tool. Across trials, information is either from trials at a low risk of bias (green), or from trials at unclear risk of bias (yellow), or from trials at high risk of bias (red). For each study, every bias domain will be checked, the given summary represents an assessment of bias risk across studies. For each bias domain, low risk of bias means that information is from studies at low risk of bias, high risk of bias indicates the proportion of information from studies at high risk of bias which might be sufficient to affect the interpretation of the results, and unclear risk of bias refers to information from studies at low or unclear risk of bias.

any inconsistency: $\mathrm{I}^{2}=[(\mathrm{Q}-\mathrm{df})] / \mathrm{Q} \times 100 \%$, where $\mathrm{Q}$ is the $\chi^{2}$ statistic and $\mathrm{df}$ is its degrees of freedom. A value for $\mathrm{I}^{2}>50 \%$ was considered to represent substantial heterogeneity. ${ }^{17}$ To consider heterogeneity, the random-effects model was used to estimate RRs and MDs with 95\% CIs. Forest plots were generated to illustrate the study-specific effect sizes along with a $95 \%$ CI.

A random-effects univariate meta-regression was performed to examine the association between the change in percentage energy from SFA, PUFA (mixed n- 6 and $\mathrm{n}-3)$, MUFA, as well as linoleic acid in the interventions versus control groups, and the dependent variables (log change RRs for all-cause mortality, CVD mortality, cardiovascular events and myocardial infarction). Furthermore, multivariate analyses were performed including all dietary fatty acid changes in a meta-regression model. As reported previously by Mensink et $a l^{5}$, effects of protein (available only for five studies) and alcohol could not be estimated. The $\mathrm{p}$ values for differences in effects between the covariates were obtained using the metareg function of STATA V.12.0 (Stata-Corp, College Station, Texas, USA). Two sided $\mathrm{p}$ values $<0.05$ were considered to be statistically significant. To determine the presence of publication bias, the symmetry of the funnel plots was assessed in which mean differences were plotted against their corresponding SEs. Sensitivity analyses were carried out to evaluate the influence of single trials on each meta-regression result. In addition, sensitivity analyses for PUFA versus SFA trials focusing on secondary prevention and sensitivity analysis for trials adopting 'fish advice' versus 'no fish advice' and combining reduced and modified fat diets were performed.

The quality of evidence was assessed according to the Grading of Recommendations Assessment, Development and Evaluation (GRADE) guidelines. ${ }^{18} 19$

\section{Missing data}

Exposure data (SFA, PUFA, MUFA) for three study control groups ${ }^{20-22}$ were imputed based on average background dietary intakes in similar populations at that time period, the corresponding data were derived from the National Diet Heart Study control group. ${ }^{23}$ For evaluating linoleic acid, all of the trials that reported on total PUFA were included. Except for the Lyon Diet Heart Study, none of these studies had a major focus on n-3 fatty acids. Therefore, total PUFA in each of these other trials would be nearly all $(90 \%+)$ linoleic acid. For studies giving information on the type of vegetable oil used, the proportion of linoleic acid and $\alpha$-linolenic acid in total PUFA could be directly calculated.

\section{RESULTS}

\section{Study characteristics}

Altogether, 12 studies extracted from 2059 articles met the inclusion criteria and were included in the quantitative analysis. $^{20-22}$ 24-33 The study by Singh et $a l^{31}$ was included only in the sensitivity analysis, since this publication has been questioned for veracity. ${ }^{34} 35$ The detailed steps of the meta-analysis/meta-regression article selection process are described as a flow diagram in the online supplementary figure S1.

All studies included were RCTs with a duration ranging between 12 months and 6 years, published between 1965 and 2013 and enrolling a total of 6744 participants (7150 including the Singh trial). General and specific study characteristics are summarised in table 1 and online supplementary table $S 1$, respectively.

All studies included participants with established CHD. Some trials had to be excluded due to various reasons: one study enrolled participants at high risk of CHD with only $50 \%$ of its participants suffering from $\mathrm{CAD}^{36}$; another trial was not randomised ${ }^{37}$; a recently performed secondary prevention RCT comparing a Mediterranean versus a low fat diet did not fulfil the inclusion criteria, since the intervention groups were not distinguished with respect to SFA intake ${ }^{38}$; all secondary prevention studies with multifactorial 
intervention protocols (eg, smoking cessation, better drug control, stress management or exercise) were excluded as well. ${ }^{39-44}$

In the reduced/modified diet groups, the range for SFA varied between $7.2 \%$ and $14 \%$, while the respective values in the control group varied between $11.7 \%$ and $26.4 \%$. PUFA intakes were in the range $5-20.9 \%$, MUFA ranged between $8 \%$ and $26 \%$, and linoleic acid intake was at in the range 3.6-19.7\% in the reduced/modified fat diet groups, respectively. Results of the univariate and multivariate meta-regression are summarised in table 2.

\section{Reduced versus control diets; modified fat versus control diets}

With respect to clinical endpoints, no significant risk reduction could be observed considering all-cause mortality (RR 0.79 (95\% CI 0.42 to 1.48 ), $\mathrm{p}=0.47 ; \mathrm{I}^{2}=0 \%$ ) and cardiovascular mortality (RR $0.93(95 \%$ CI 0.66 to 1.31), $\left.\mathrm{p}=0.66 ; \mathrm{I}^{2}=0 \%\right)$, combined cardiovascular events (RR 0.93 (95\% CI 0.65 to 1.34 ), $\mathrm{p}=0.71 ; \mathrm{I}^{2}=57 \%$ ) and myocardial infarction (RR 1.18 (95\% CI 0.88 to 1.59 ), $\left.\mathrm{p}=0.26 ; \mathrm{I}^{2}=19 \%\right)$ comparing reduced fat versus control diets (see online supplementary figures S2-S5). Furthermore comparing modified fat versus control diets showed no significant effects on all-cause mortality (RR 0.92 (95\% CI 0.68 to 1.25$), \mathrm{p}=0.60 ; \mathrm{I}^{2}=59 \%$ ) and cardiovascular mortality (RR 0.96 (95\% CI 0.65 to 1.42 ), $\mathrm{p}=0.84 ; \mathrm{I}^{2}=69 \%$ ), combined cardiovascular events (RR 0.85 (95\% CI 0.63 to 1.15$\left.), \mathrm{p}=0.30 ; \mathrm{I}^{2}=75 \%\right)$ and myocardial infarction (RR 0.76 (95\% CI 0.54 to 1.09), $\mathrm{p}=0.13$; $\mathrm{I}^{2}=55 \%$ ) could be observed (see online supplementary figures S6-S9). Pooling reduced and modified fat diets all together resulted in no significant changes (see online supplementary figures S10-S13).

\section{Univariate meta-regression}

Taken together, the univariate meta-regression showed no significant association between changes in SFA, PUFA, MUFA, linoleic acid and risk of all-cause mortality, cardiovascular mortality, total cardiovascular events and myocardial infarction (see online supplementary figures S14-S29).

\section{Multivariate meta-regression}

Similar to the univariate model, the multivariate meta-regression did not reveal any significant association between changes in SFA, PUFA and MUFA and risk of all-cause mortality, cardiovascular mortality, cardiovascular events and myocardial infarction.

\section{Sensitivity analyses}

Sensitivity analyses were performed to evaluate the influence of single trials on each meta-regression. None of the trials had a significant impact on the results of the univariate and multivariate meta-regression.

An additional sensitivity analysis was carried out according to the main analysis of Mozaffarian et at as well as Skeaff and Miller, ${ }^{6}$ evaluating the replacement of
SFA by PUFA (including the new data of the Sydney Diet Heart Study ${ }^{33}$ ). The results showed that replacing SFA by PUFA was not associated with a significant risk reduction for all-cause mortality, cardiovascular mortality, combined cardiovascular events and myocardial infarction (see online supplementary figures S30-S33) in participants with established CHD/CAD.

Another sensitivity analysis was performed including only those trials recommending a higher consumption of fatty fish. This resulted in a significant reduction of cardiovascular events, cardiovascular mortality and allcause mortality (see online supplementary figures S34S36).

Since in the Sydney Diet Heart Study a commercial margarine probably high in transfatty acids was used to deliver PUFA to the intervention group, a sensitivity analysis was performed excluding this trial. However, all results of the primary analysis could be confirmed.

\section{Publication bias}

The funnel plots indicate moderate asymmetry, suggesting that publication bias cannot be completely excluded as a factor of influence on the present meta-analysis (see online supplementary figures S37-S40).

\section{Overall quality of evidence}

The overall quality of evidence rated according to the GRADE guidelines for all outcomes was moderate.

\section{DISCUSSION}

In this systematic review of 7150 participants with established CHD or CAD comparing reduced and/or modified fat diets versus control diets, no significant risk reduction (moderate quality of evidence) for all-cause mortality, cardiovascular mortality, cardiovascular events and myocardial infarction could be observed. Since no previous meta-analysis compared the effects of reduced and/or modified fat diets as a means for secondary prevention, the present data will be discussed together with results of combined primary/secondary prevention trials.

Small but nevertheless significant reductions in cardiovascular events could be observed by Hooper et $a t^{45}$ as well as Truswell ${ }^{46}$ following their respective meta-analyses of intervention studies investigating the effects of a modification of fatty acid intake as a secondary preventive measure in patients with CVD. Systematic reviews analysing trials targeted both at primary and secondary prevention found that replacing SFA with unsaturated fatty acids reduced cardiovascular events. ${ }^{11}$ The question whether these benefits are due to CHO, MUFA or due to PUFA is discussed controversially. The systematic review by Skeaff and Miller observed a significant increase in risk (by $25 \%$ ) of CHD death in the highest category of dietary PUFA. In contrast, pooling RCTs indicated that a $5 \%$ increase in PUFA intake was associated with a significant reduction in CHD events. ${ }^{6}$ Mente et $a l^{47}$ observed a 
Table 1 General study characteristics

\begin{tabular}{|c|c|c|c|c|c|c|c|c|c|c|c|}
\hline Reference & $\begin{array}{l}\text { Sample } \\
\text { size, } \\
\text { baseline } \\
\text { BMI (kg/ } \\
\text { m²) }^{2}\end{array}$ & $\begin{array}{l}\text { Age } \\
\text { (years), } \\
\text { female } \\
(\%) \\
\text { male } \\
(\%)\end{array}$ & $\begin{array}{l}\text { Duration } \\
\text { (years) }\end{array}$ & $\begin{array}{l}\text { SFA/PUFA/ } \\
\text { MUFA/trans } \\
\text { FA (as } \\
\text { indicated by } \\
\text { the } \\
\text { investigators) }\end{array}$ & LA, ALA & $\begin{array}{l}\text { Fish } \\
\text { consumption } \\
\text { advice }\end{array}$ & $\begin{array}{l}\text { TF, } \\
\text { cholesterol }\end{array}$ & $\begin{array}{l}\text { Inclusion } \\
\text { criteria }\end{array}$ & Supplement & $\begin{array}{l}\text { Energy } \\
\text { amount } \\
\text { (end of } \\
\text { the } \\
\text { study) }\end{array}$ & Outcomes \\
\hline $\begin{array}{l}\text { Ball et al } \\
(1965)^{20 *}\end{array}$ & $\begin{array}{l}264 \\
\text { nd }\end{array}$ & $\begin{array}{l}<65 \\
100 \%\end{array}$ & 4 & $\begin{array}{l}\text { I: } 9 \%, 5 \%, 8 \% \\
\text { C: } 16-18 \% \text {, } \\
7 \%, 15 \%\end{array}$ & $\begin{array}{l}\text { I: } 4.5 \% \\
\text { C: } 6.3 \%\end{array}$ & & $\begin{array}{l}\mathrm{I}: 22 \% \text {, } \\
330 \mathrm{mg} \\
\mathrm{C:} 47 \% \text {, } \\
650- \\
750 \mathrm{mg}\end{array}$ & Post-MI & - & $\begin{array}{l}2030 \\
2360\end{array}$ & $\begin{array}{l}\text { ACM, } \\
\text { CVM, } \\
\text { CVE, MI }\end{array}$ \\
\hline $\begin{array}{l}\text { Burr et al } \\
(1989)^{27}\end{array}$ & 2033 & $\begin{array}{l}56.6 \\
100 \%\end{array}$ & 2 & $\begin{array}{l}\text { I: } 11.3 \%, 8.9 \% \text {, } \\
12.1 \% \\
\text { C: } 15 \%, 6.4 \% \text {, } \\
13.6 \%\end{array}$ & $\begin{array}{l}\text { I: } 8.01 \% \\
\text { C: } 5.76 \%\end{array}$ & & $\begin{array}{l}\text { I: } 32.3 \% \\
\text { C: } 35 \% \text {, } \\
650- \\
750 \mathrm{mg}\end{array}$ & Post-MI & - & nd & $\begin{array}{l}\text { ACM, } \\
\text { CVM, CVE }\end{array}$ \\
\hline $\begin{array}{l}\text { de Lorgeril } \\
\text { et al }(1994)^{24}\end{array}$ & $\begin{array}{l}605 \\
\text { nd }\end{array}$ & $\begin{array}{l}53.5 \\
9.25 \% \\
90.75 \%\end{array}$ & 4 & $\begin{array}{l}\text { I: } 8.3 \%, 8.2 \% \text {, } \\
12.9 \% \\
\text { C: } 11.7 \% \\
11.4 \%, 10.3 \%\end{array}$ & $\begin{array}{l}\mathrm{I}: 3.6 \%, 0.81 \% \\
\text { C: } 5.3 \% \\
0.27 \%\end{array}$ & yes & $\begin{array}{l}\text { I: } 30.5 \% \text {, } \\
217 \\
\text { C: } 32.7 \% \text {, } \\
318 \mathrm{mg}\end{array}$ & Post-MI & $\begin{array}{l}\text { I: Canola oil } \\
\text { based } \\
\text { margarine }\end{array}$ & $\begin{array}{l}1928 \\
2140\end{array}$ & $\begin{array}{l}\text { ACM, } \\
\text { CVM, CVE }\end{array}$ \\
\hline $\begin{array}{l}\text { Howard et al } \\
(2006)^{32}\end{array}$ & $\begin{array}{l}2277 \\
29.1\end{array}$ & $\begin{array}{l}62.3 \\
100 \%\end{array}$ & 6 & $\begin{array}{l}\text { I: } 9.5 \%, 6.1 \% \text {, } \\
10.8 \%, 1.8 \% \\
\text { C: } 12.4 \% \\
7.5 \%, 14.2 \% \\
2.4 \%\end{array}$ & $\begin{array}{l}\text { I: } 5.49 \% \\
\text { C: } 6.75 \%\end{array}$ & & $\begin{array}{l}\text { I: } 28.8 \% \text {, } \\
193.6 \mathrm{mg} \\
\text { C: } 37 \% \text {, } \\
243.5 \mathrm{mg}\end{array}$ & $\begin{array}{l}\text { Post-MI, } \\
\text { stroke, } \\
\text { CABG or } \\
\text { PCl }\end{array}$ & - & $\begin{array}{l}1431 \\
1546\end{array}$ & $\begin{array}{l}\text { CVM, } \\
\text { CVE, MI, } \\
\text { stroke }\end{array}$ \\
\hline $\begin{array}{l}\text { Leren et al } \\
(1970)^{21 *}\end{array}$ & $\begin{array}{l}412 \\
\text { nd }\end{array}$ & $\begin{array}{l}56.25 \\
100 \%\end{array}$ & 5 & $\begin{array}{l}\text { I: } 8.5 \%, 20.7 \% \text {, } \\
10.1 \% \\
\text { C: } 16-18 \% \\
7 \%, 15 \%\end{array}$ & $\begin{array}{l}\text { I: } 14.8 \%, 2.7 \% \\
\text { C: } 3.3 \%, \text { nd }\end{array}$ & yes & $\begin{array}{l}\text { I: } 39 \% \\
264 \mathrm{mg} \\
\mathrm{C:} 40 \% \text {, } \\
650- \\
750 \mathrm{mg}\end{array}$ & Post-MI & $\begin{array}{l}\text { I: } 75 \mathrm{~g} \\
\text { Soya-bean } \\
\text { oil }\end{array}$ & 2380 & $\begin{array}{l}\text { ACM, } \\
\text { CVM, } \\
\text { CVE, MI }\end{array}$ \\
\hline $\begin{array}{l}\text { MRC } \\
(1968)^{26}\end{array}$ & $\begin{array}{l}393 \\
\text { nd }\end{array}$ & $\begin{array}{l}53.5 \\
100 \%\end{array}$ & 5 & $\begin{array}{l}\text { I: } 11.3 \% \text {, } \\
20.4 \%, 14.3 \% \\
\text { C: } 26.4 \% \text {, } \\
4.4 \%, 12.2 \%\end{array}$ & $\begin{array}{l}\mathrm{I}: 16.3 \%, 2.3 \% \\
\mathrm{C}: 3.96\end{array}$ & & $\begin{array}{l}\text { I: } 46 \% \\
\text { C: } 43 \% \text {, } \\
650- \\
750 \mathrm{mg}\end{array}$ & Post-MI & $\begin{array}{l}\text { I: } 85 \mathrm{~g} \\
\text { soya-bean } \\
\text { oil }\end{array}$ & $\begin{array}{l}\text { nd } \\
\text { nd }\end{array}$ & $\begin{array}{l}\text { ACM, } \\
\text { CVM, } \\
\text { CVE, MI }\end{array}$ \\
\hline $\begin{array}{l}\text { Michalsen } \\
\text { et al }(2006)^{29}\end{array}$ & $\begin{array}{l}101 \\
26.55\end{array}$ & $\begin{array}{l}59.4 \\
23 \% \\
77 \%\end{array}$ & 1 & $\begin{array}{l}\text { I: } 10.1 \%, 6.1 \% \text {, } \\
10.6 \% \\
\text { C: } 13 \%, 7.4 \% \text {, } \\
12.2 \%\end{array}$ & $\begin{array}{l}\mathrm{I}: 4.8 \%, 0.72 \% \\
\mathrm{C}: 6.2 \%, 0.7 \%\end{array}$ & yes & $\begin{array}{l}\text { I: } 32.2 \% \text {, } \\
251 \\
\text { C: } 35.2 \% \text {, } \\
265 \mathrm{mg}\end{array}$ & $\begin{array}{l}\text { CAD verified } \\
\text { by coronary } \\
\text { angiography }\end{array}$ & - & $\begin{array}{l}2241 \\
2237\end{array}$ & - \\
\hline $\begin{array}{l}\text { Rose et al } \\
(1965)^{\star 22}\end{array}$ & $\begin{array}{l}80 \\
\text { nd }\end{array}$ & $\begin{array}{l}<70 \\
100 \%\end{array}$ & 2 & $\begin{array}{l}\text { I: instructed to } \\
\text { avoid fried } \\
\text { foods, fatty } \\
\text { meats, } \\
\text { sausages, } \\
\text { pastry, }\end{array}$ & $\begin{array}{l}\text { I-olive: } 8.2 \% \text {, } \\
\text { 0.28\% } \\
\text { I-corn: } 19.7 \% \text {, } \\
0.07 \% \\
\text { C: } 6.3 \%\end{array}$ & & $\begin{array}{l}\text { I-olive: } \\
46.2 \% \\
\text { I-corn: } \\
50.5 \% \\
\text { C: } 32.5 \%\end{array}$ & $\begin{array}{l}\text { Post-MI, } \\
\text { angina } \\
\text { pectoris }\end{array}$ & $\begin{array}{l}\text { I: } 80 \text { g olive } \\
\text { oil; } \\
80 \mathrm{~g} \text { corn oil }\end{array}$ & $\begin{array}{l}2045 \\
2070 \\
1933\end{array}$ & $\begin{array}{l}\text { ACM, } \\
\text { CVM, } \\
\text { CVE, MI }\end{array}$ \\
\hline
\end{tabular}


Table 1 Continued

\begin{tabular}{|c|c|c|c|c|c|c|c|c|c|c|c|}
\hline Reference & $\begin{array}{l}\text { Sample } \\
\text { size, } \\
\text { baseline } \\
\text { BMI (kg/ } \\
\text { m²) }^{2}\end{array}$ & $\begin{array}{l}\text { Age } \\
\text { (years), } \\
\text { female } \\
(\%) \\
\text { male } \\
(\%)\end{array}$ & $\begin{array}{l}\text { Duration } \\
\text { (years) }\end{array}$ & $\begin{array}{l}\text { SFA/PUFA/ } \\
\text { MUFA/trans } \\
\text { FA (as } \\
\text { indicated by } \\
\text { the } \\
\text { investigators) }\end{array}$ & LA, ALA & $\begin{array}{l}\text { Fish } \\
\text { consumption } \\
\text { advice }\end{array}$ & $\begin{array}{l}\text { TF, } \\
\text { cholesterol }\end{array}$ & $\begin{array}{l}\text { Inclusion } \\
\text { criteria }\end{array}$ & Supplement & $\begin{array}{l}\text { Energy } \\
\text { amount } \\
\text { (end of } \\
\text { the } \\
\text { study) }\end{array}$ & Outcomes \\
\hline & & & & $\begin{array}{l}\text { ice-cream, } \\
\text { cheese, cakes, } \\
\text { butter, milk, } \\
\text { eggs restricted } \\
\text { I-olive: } 11.7 \% \text {, } \\
8.7 \%, 26 \% \\
\text { I-corn: } 14 \% \text {, } \\
21.85 \%, 14.9 \% \\
\text { C: no advice } \\
\text { on dietary fat } \\
\text { C: } 16-18 \%, \\
7 \%, 7 \%\end{array}$ & & & & & & & \\
\hline $\begin{array}{l}\text { Singh et al } \\
(1992)^{31}\end{array}$ & $\begin{array}{l}406 \\
23.8\end{array}$ & $\begin{array}{l}51.25 \\
12 \% \\
88 \%\end{array}$ & 1 & $\begin{array}{l}\text { I: } 7.2 \%, 8.6 \% \text {, } \\
8 \% \\
\text { C: } 10.8 \%, 7 \% \text {, } \\
10.2 \%\end{array}$ & $\begin{array}{l}\text { I: } 7.74 \% \\
\text { C: } 6.3 \%\end{array}$ & yes & $\begin{array}{l}\text { I: } 23.8 \% \\
\text { C: } 28 \%\end{array}$ & $\begin{array}{l}\text { Post-MI, } \\
\text { unstable } \\
\text { angina } \\
\text { pectoris }\end{array}$ & - & $\begin{array}{l}1810 \\
1940\end{array}$ & $\begin{array}{l}\text { ACM, } \\
\text { CVM, } \\
\text { CVE, MI }\end{array}$ \\
\hline $\begin{array}{l}\text { Sondergaard } \\
\text { et al (2003) }\end{array}$ & $\begin{array}{l}131 \\
\text { nd }\end{array}$ & $\begin{array}{l}62.5 \\
30 \% \\
70 \%\end{array}$ & 1 & $\begin{array}{l}\text { I: } 10.3 \%, 7.5 \% \text {, } \\
8.4 \% \\
\text { C: } 12 \%, 6.8 \% \text {, } \\
10.1 \%\end{array}$ & $\begin{array}{l}\text { I: } 6.75 \% \\
\text { C: } 6.3 \%\end{array}$ & yes & $\begin{array}{l}\text { I: } 26.2 \% \\
\text { C: } 28.9 \%\end{array}$ & $\begin{array}{l}\text { Post-MI, } \\
\text { stable/ } \\
\text { unstable } \\
\text { angina } \\
\text { pectoris }\end{array}$ & - & $\begin{array}{l}1520 \\
1550\end{array}$ & - \\
\hline $\begin{array}{l}\text { Watts et al } \\
(1992)^{28}\end{array}$ & $\begin{array}{l}90 \\
26.25\end{array}$ & $\begin{array}{l}51.4 \\
100 \%\end{array}$ & 3 & $\begin{array}{l}\text { I: } 8-10 \%, 8 \% \text {, } \\
10 \%, 1.08 \% \\
\text { C: } 17 \%, \\
5.2 \%, 14.8 \%, \\
1.8 \%\end{array}$ & $\begin{array}{l}\text { I: } 5 \%, 0.32 \% \\
\text { C: } 3.9 \% \\
0.41 \%\end{array}$ & & $\begin{array}{l}\text { I: } 27 \% \\
\text { C: } 37 \%\end{array}$ & $\begin{array}{l}\text { Angina } \\
\text { pectoris }\end{array}$ & - & nd & $\begin{array}{l}\text { ACM, } \\
\text { CVM, } \\
\text { CVE, MI }\end{array}$ \\
\hline $\begin{array}{l}\text { Woodhill et al } \\
(1978)^{25} \\
\text { Ramsden } \\
\text { et al }(2013)^{33}\end{array}$ & $\begin{array}{l}458 \\
\text { nd }\end{array}$ & $\begin{array}{l}49 \\
100 \%\end{array}$ & 5 & $\begin{array}{l}\text { I: } 9.8 \%, 15.2 \% \text {, } \\
11.5 \% \\
\text { C: } 13.5 \% \text {, } \\
8.9 \%, 13.8 \%\end{array}$ & $\begin{array}{l}\text { I: } 15 \%, \text { nd } \\
\text { C: } 8 \%, \text { nd }\end{array}$ & & $\begin{array}{l}\text { I: } 38.3 \% \text {, } \\
248 \\
\text { C: } 38.1 \% \text {, } \\
342\end{array}$ & $\begin{array}{l}\text { Post-MI, } \\
\text { angina } \\
\text { pectoris, } \\
\text { coronary } \\
\text { insufficiency }\end{array}$ & - & $\begin{array}{l}2289 \\
2191\end{array}$ & $\begin{array}{l}\text { ACM, } \\
\text { CVM, CVE }\end{array}$ \\
\hline
\end{tabular}

*SFA, MUFA and PUFA for study control groups were imputed based on average background dietary intakes in similar populations of the Diet Heart Study control group of $1968{ }^{26}$

ACM, all-cause mortality; ALA, $\alpha$-linolenic acid; BMI, body mass index; C, control; CABG, coronary artery bypass graft ,CAD, coronary artery disease; CVE, cardiovascular events; CVM,

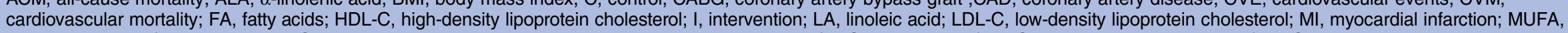

monounsaturated fat; nd, no data; PCI, percutaneous coronary intervention; PUFA, polyunsaturated fat; SFA, saturated fat; TC, total cholesterol; TF, total fat; TG, triacylglycerols. 
Table 2 Univariate and multivariate meta-regression for change in dietary fatty acid (covariate=percentage energy change in SFA, PUFA, MUFA and LA between intervention vs control groups)

\begin{tabular}{|c|c|c|c|c|c|}
\hline Covariate & $\begin{array}{l}\text { Number of studies } \\
\text { or subsets }\end{array}$ & $\beta$-Coefficient & $95 \% \mathrm{Cl}$ & p Value & Heterogeneity $\left(\mathrm{I}^{2}\right)(\%)$ \\
\hline & \multicolumn{5}{|c|}{ All-cause mortality (univariate) } \\
\hline SFA & 8 & 0.0029 & -0.1156 to 0.1216 & 0.953 & 75.24 \\
\hline PUFA & 8 & 0.0253 & -0.0351 to 0.0858 & 0.345 & 72.97 \\
\hline MUFA & 8 & -0.0024 & -0.1475 to 0.1426 & 0.968 & 72.04 \\
\hline \multirow[t]{2}{*}{ LA } & 8 & 0.0355 & -0.0387 to 0.1099 & 0.286 & 73.04 \\
\hline & \multicolumn{5}{|c|}{ Cardiovascular mortality (univariate) } \\
\hline SFA & 9 & 0.0089 & -0.0900 to 0.1079 & 0.836 & 56.89 \\
\hline PUFA & 9 & 0.0182 & -0.0449 to 0.0813 & 0.517 & 58.23 \\
\hline MUFA & 9 & 0.0186 & -0.1428 to 0.1801 & 0.793 & 56.58 \\
\hline \multirow[t]{2}{*}{ LA } & 9 & 0.0245 & -0.0517 to 0.1008 & 0.472 & 58.10 \\
\hline & \multicolumn{5}{|c|}{ Total cardiovascular events (univariate) } \\
\hline SFA & 9 & 0.0181 & -0.0884 to 0.1247 & 0.699 & 70.88 \\
\hline PUFA & 9 & 0.0157 & -0.0444 to 0.0759 & 0.556 & 73.70 \\
\hline MUFA & 9 & -0.0069 & -0.1172 to 0.1033 & 0.885 & 75.80 \\
\hline \multirow[t]{2}{*}{ LA } & 9 & 0.0224 & -0.0492 to 0.0940 & 0.484 & 74.05 \\
\hline & \multicolumn{5}{|c|}{ Myocardial infarction (univariate) } \\
\hline SFA & 9 & 0.0114 & -0.0813 to 0.1041 & 0.780 & 66.23 \\
\hline PUFA & 9 & 0.0011 & -0.0529 to 0.0551 & 0.962 & 66.60 \\
\hline MUFA & 9 & -0.0224 & -0.1267 to 0.0818 & 0.627 & 69.19 \\
\hline \multirow[t]{2}{*}{ LA } & 9 & 0.0028 & -0.0627 to 0.06840 & 0.922 & 66.87 \\
\hline & \multicolumn{5}{|c|}{ All-cause mortality (multivariate) } \\
\hline SFA & 8 & 0.0800 & -0.1390 to 0.2991 & 0.368 & 77.39 \\
\hline PUFA & 8 & 0.0568 & -0.0534 to 0.1671 & 0.226 & \\
\hline \multirow[t]{2}{*}{ MUFA } & 8 & -0.0230 & -0.2254 to 0.1793 & 0.768 & \\
\hline & \multicolumn{5}{|c|}{ Cardiovascular mortality (multivariate) } \\
\hline SFA & 9 & 0.0768 & -0.1422 to 0.2959 & 0.409 & 67.14 \\
\hline PUFA & 9 & 0.0584 & -0.0660 to 0.1830 & 0.281 & \\
\hline \multirow[t]{2}{*}{ MUFA } & 9 & -0.0381 & -0.2691 to 0.1927 & 0.689 & \\
\hline & \multicolumn{5}{|c|}{ Total cardiovascular events (multivariate) } \\
\hline SFA & 9 & 0.1103 & -0.0691 to 0.2898 & 0.175 & 77.96 \\
\hline PUFA & 9 & 0.0638 & -0.0347 to 0.1623 & 0.157 & \\
\hline \multirow[t]{2}{*}{ MUFA } & 9 & -0.0825 & -0.2417 to 0.0765 & 0.240 & \\
\hline & \multicolumn{5}{|c|}{ Myocardial infarction (multivariate) } \\
\hline SFA & 9 & 0.0665 & -0.1237 to 0.2568 & 0.410 & 75.28 \\
\hline PUFA & 9 & 0.0355 & -0.0703 to 0.1414 & 0.428 & \\
\hline MUFA & 9 & -0.0675 & -0.2424 to 0.1072 & 0.366 & \\
\hline
\end{tabular}

significant inverse correlation between MUFA (but not PUFA) intake and CHD events, thus favouring MUFA as a mediator for the beneficial effects of reduced SFA intake. Furthermore, a review of 16 meta-analyses indicated that a diet rich in MUFA has several beneficial effects on a broad range of CVD risk factors, in the primary prevention of CVD. ${ }^{48}$

In contrast to the data presented in this systematic review, a recent meta-analysis of observational studies suggests that replacing SFAs with PUFAs may have a greater benefit than replacing SFAs with $\mathrm{CHO}$ or MUFA. $^{8}$ Moreover, the meta-analysis of RTCs by Mozaffarian et a ${ }^{\ominus}$ reported a significant reduction in CHD events (by 19\%) following the replacement of SFA by PUFA. However, adapting (for secondary prevention) and updating (including new data from the Sydney Diet Heart Study) the meta-analysis by Mozaffarian et $a l^{9}{ }^{9}$ as was carried out in a sensitivity analysis investigating the replacement of SFA by PUFA in the present study, resulted in neither beneficial nor detrimental effects on all outcome parameters. Compared with meta-analyses of observational studies, those of RCTs are considered to have a higher grade of quality. ${ }^{18}$ RCTs of lifestyle behaviours such as diet are often limited by lack of double blinding, non-compliance, cross-over and dropout-as evidenced by the trials in the current meta-analysis-so that well-designed analyses in prospective cohort studies provide important evidence with complementary strengths and limitations.

No detrimental effects of increased amounts of linoleic acid could be observed in meta-regression. The results of the present meta-regression are in discrepancy with the observations of a recent meta-analysis of Ramsden et $a l,{ }^{33}$ providing evidence that replacement of 
SFA with linoleic acid was associated with increased rates of death from all causes, CHD and CVD, respectively. In order to rationalise their results, Ramsden et al proposed a mechanistic model linking dietary linoleic acid to CVD pathogenesis. It is proposed that diets high in n-6 linoleic acid facilitate production of oxidised linoleic acid metabolites mediating progression of atherosclerosis and thus leading to higher rates of cardiovascular mortality. ${ }^{33}$ Owing to the low number of studies available and the inherent biases of the method, the findings of the present meta-regressions must be interpreted in a very conservative manner. However, when using these results to generate a hypothesis, it still seems reasonable to replace SFA by PUFA in the secondary prevention of CHD, although SFA should not be completely substituted by $n-6$ fatty acids as recommended by the FAO. ${ }^{7}$ Instead, dietary advice should rather focus on increasing the uptake of n-3 fatty acids, predominantly in the form of fatty fish.

The present systematic review does not consider unpublished data, and it cannot be excluded that these results may have had at least a moderate impact on the effect size estimates. Examination of funnel plots showed little to moderate asymmetry suggesting that publication bias cannot be completely excluded as a confounder of the present meta-analysis (eg, it remains possible that small studies yielding inconclusive data have not been published). Another limitation of nutritional intervention trials is the heterogeneity of various aspects and characteristics of the study protocols. RCTs in the present analysis varied with respect to the type(s) of diets used (eg, advised to supplement various oils, fatty fish), and size of study population. Some of the included studies date back to 50 years, and not all of the studies provided information on the quality of their respective setup (eg, method of randomisation, follow-up protocol with reasons for withdrawal, see figure 1 for risk of bias assessment according to the Cochrane Collaboration), again demanding a conservative interpretation of results. Another potential limitation of the present meta-analysis is rather specifically related to the research question. Total fat intake in the reduced and/or modified diet groups ranged between $20 \%$ and $50 \%$ of TEC. In some trials, dietary fat intake was established by adding corn, olive or soybean oil, ${ }^{21} 26$ while others implemented canola oil-based margarine. ${ }^{24}$ In several trials, the participants in the reduced and/or modified fat groups were provided with additional dietary advice such as to increase the consumption of fatty fish, ${ }^{21} 24$ 29-31 vegetables and fruits, ${ }^{24-31}$ or nuts ${ }^{31}$ as well as to pay attention to their cholesterol intake. In contrast to these heterogeneous aspects of the study designs, length of trials included in this systematic review was rather homogenous with all RCTs having a running time of at least 1 year. However, this might be interconnected with another major limitation of dietary intervention trials, that is, the issue of compliance. Participants in the different intervention groups may not exactly adhere to the advised or prescribed dietary protocol. In the end, this may not only lead to deviations from the target values but may result in only minimal differences between the study arms.

\section{CONCLUSION}

The present meta-analyses and meta-regressions provide no evidence for a beneficial secondary preventive effect of either reduced and/or modified fat diets or replacement of SFA by PUFA in participants with established CVD. Although pharmaceutical treatment via lipid-lowering medications is considered to be an effective therapeutic measure for patients with established CVD, many international authorities recommend modifications of fat intake with a special emphasis on SFA as well. ${ }^{13} 4950$ The current AHA/ACC guidelines promote to reduce the total fat intake to less than $35 \%$ of TEC and SFA intake to less than $7 \%$ of TEC, respectively. However, recommending higher intakes of PUFA in general instead of SFA might not be appropriate. Sensitivity analysis indicates that it seems reasonable to modify this general recommendation by promoting higher dietary n-3 PUFA as a substitute for SFA.

Contributors LS and GH were involved in the conception and design of the study, involved in the collection of data, statistical analysis, interpretation of data and drafting of the article. LS and GH approved the final version of the manuscript.

Funding This research received no specific grant from any funding agency in the public, commercial or not-for-profit sectors.

Competing interests None.

Provenance and peer review Not commissioned; externally peer reviewed.

Data sharing statement No additional data are available.

Open Access This is an Open Access article distributed in accordance with the Creative Commons Attribution Non Commercial (CC BY-NC 3.0) license, which permits others to distribute, remix, adapt, build upon this work noncommercially, and license their derivative works on different terms, provided the original work is properly cited and the use is non-commercial. See: http:// creativecommons.org/licenses/by-nc/3.0/

\section{REFERENCES}

1. Hegsted DM, McGandy RB, Myers ML, et al. Quantitative effects of dietary fat on serum cholesterol in man. Am J Clin Nutr 1965;17:281-95.

2. Keys A, Mickelsen O, Miller EO, et al. The relation in man between cholesterol levels in the diet and in the blood. Science 1950;112:79-81.

3. Keys A, Menotti A, Karvonen MJ, et al. The diet and 15-year death rate in the seven countries study. Am J Epidemiol 1986;124:903-15.

4. Siri-Tarino PW, Sun Q, Hu FB, et al. Meta-analysis of prospective cohort studies evaluating the association of saturated fat with cardiovascular disease. Am J Clin Nutr 2010;91:535-46.

5. Mensink RP, Zock PL, Kester AD, et al. Effects of dietary fatty acids and carbohydrates on the ratio of serum total to HDL cholesterol and on serum lipids and apolipoproteins: a meta-analysis of 60 controlled trials. Am J Clin Nutr 2003;77:1146-55.

6. Skeaff CM, Miller J. Dietary fat and coronary heart disease: summary of evidence from prospective cohort and randomised controlled trials. Ann Nutr Metab 2009;55:173-201.

7. FAO. Fats and fatty acids in human nutrition. Report of an Expert Consultation. 2010.

8. Jakobsen MU, O'Reilly EJ, Heitmann BL, et al. Major types of dietary fat and risk of coronary heart disease: a pooled analysis of 11 cohort studies. Am J Clin Nutr 2009;89:1425-32.

9. Mozaffarian D, Micha R, Wallace S. Effects on coronary heart disease of increasing polyunsaturated fat in place of saturated fat: a 
systematic review and meta-analysis of randomized controlled trials. PLoS Med 2010;7:e1000252.

10. Hooper L, Summerbell CD, Higgins JP, et al. Dietary fat intake and prevention of cardiovascular disease: systematic review. BMJ 2001;322:757-63.

11. Hooper L, Summerbell CD, Thompson R, et al. Reduced or modified dietary fat for preventing cardiovascular disease. Cochrane Database Syst Rev 2012;5:CD002137.

12. USDA. US department of health and human services: dietary guidelines for Americans, 2010. Washington: US Government Printing Office, 2010.

13. Smith SC Jr, Benjamin EJ, Bonow RO, et al. AHA/ACCF secondary prevention and risk reduction therapy for patients with coronary and other atherosclerotic vascular disease: 2011 update: a guideline from the American Heart Association and American College of Cardiology Foundation. Circulation 2011;124:2458-73.

14. Moher D, Liberati A, Tetzlaff J, et al. Preferred reporting items for systematic reviews and meta-analyses: the PRISMA statement. PLoS Med 2009;6:e1000097.

15. Higgins JP, Altman DG, Gotzsche PC, et al. The Cochrane Collaboration's tool for assessing risk of bias in randomised trials. BMJ 2011;343:d5928.

16. Higgins JP, Green S. Cochrane Handbook of systematic reviews, Version 5.1.0 updated March 2011.

17. Higgins JP, Thompson SG, Deeks JJ, et al. Measuring inconsistency in meta-analyses. BMJ 2003;327:557-60.

18. Guyatt $G$, Oxman AD, Akl EA, et al. GRADE guidelines: 1. Introduction-GRADE evidence profiles and summary of findings tables. J Clin Epidemiol 2011;64:383-94.

19. Guyatt GH, Oxman AD, Vist GE, et al. GRADE: an emerging consensus on rating quality of evidence and strength of recommendations. BMJ 2008;336:924-6.

20. Ball KP, Hanington E, McAllen PM et al. Low-fat diet in myocardial infarction: a controlled trial. Lancet 1965;2:501-4.

21. Leren P. The Oslo diet-heart study. Eleven-year report. Circulation 1970;42:935-42.

22. Rose GA, Thomson WB, Williams RT. Corn oil in treatment of ischaemic heart disease. BMJ 1965;1:1531-3.

23. The National Diet-Heart Study Final Report. Circulation 1968;37(3 Suppl):l1-428.

24. de Lorgeril M, Renaud S, Mamelle N, et al. Mediterranean alpha-linolenic acid-rich diet in secondary prevention of coronary heart disease. Lancet 1994;343:1454-9.

25. Woodhill JM, Palmer AJ, Leelarthaepin B, et al. Low fat, low cholesterol diet in secondary prevention of coronary heart disease. Adv Exp Med Biol 1978;109:317-30.

26. Medical Research Council. Controlled trial of soya-bean oil in myocardial infarction. Lancet 1968;2:693-9.

27. Burr ML, Fehily AM, Gilbert JF, et al. Effects of changes in fat, fish, and fibre intakes on death and myocardial reinfarction: diet and reinfarction trial (DART). Lancet 1989;2:757-61.

28. Watts GF, Lewis B, Brunt JN, et al. Effects on coronary artery disease of lipid-lowering diet, or diet plus cholestyramine, in the St Thomas' Atherosclerosis Regression Study (STARS). Lancet 1992;339:563-9.

29. Michalsen A, Lehmann N, Pithan C, et al. Mediterranean diet has no effect on markers of inflammation and metabolic risk factors in patients with coronary artery disease. Eur J Clin Nutr 2006;60:478-85.

30. Sondergaard E, Moller JE, Egstrup K. Effect of dietary intervention and lipid-lowering treatment on brachial vasoreactivity in patients with ischemic heart disease and hypercholesterolemia. Am Heart $J$ 2003;145:E19.

31. Singh RB, Rastogi SS, Verma R, et al. Randomised controlled trial of cardioprotective diet in patients with recent acute myocardial infarction: results of one year follow up. BMJ 1992;304:1015-19.
32. Howard BV, Van Horn L, Hsia J, et al. Low-fat dietary pattern and risk of cardiovascular disease: the Women's Health Initiative Randomized Controlled Dietary Modification Trial. JAMA 2006;295:655-66.

33. Ramsden CE, Zamora D, Leelarthaepin B, et al. Use of dietary linoleic acid for secondary prevention of coronary heart disease and death: evaluation of recovered data from the Sydney Diet Heart Study and updated meta-analysis. BMJ 2013;346:e8707.

34. White C. Suspected research fraud: difficulties of getting at the truth. BMJ 2005;331:266.

35. Horton R. Expression of concern: Indo-Mediterranean Diet Heart Study. Lancet 2005;366:354-6.

36. Singh RB, Dubnov G, Niaz MA, et al. Effect of an Indo-Mediterranean diet on progression of coronary artery disease in high risk patients (Indo-Mediterranean Diet Heart Study): a randomised single-blind trial. Lancet 2002;360:1455-61.

37. Bierenbaum ML, Fleischman Al, Green DP, et al. The 5-year experience of modified fat diets on younger men with coronary heart disease. Circulation 1970;42:943-52.

38. Tuttle KR, Shuler LA, Packard DP, et al. Comparison of low-fat versus Mediterranean-style dietary intervention after first myocardial infarction (from The Heart Institute of Spokane Diet Intervention and Evaluation Trial). Am J Cardiol 2008;101:1523-30.

39. Ornish D, Brown SE, Scherwitz LW, et al. Can lifestyle changes reverse coronary heart disease? The Lifestyle Heart Trial. Lancet 1990;336:129-33.

40. Kallio V, Hamalainen $\mathrm{H}$, Hakkila $\mathrm{J}$, et al. Reduction in sudden deaths by a multifactorial intervention programme after acute myocardial infarction. Lancet 1979;2:1091-4.

41. Schuler G, Schlierf G, Wirth A, et al. Low-fat diet and regular, supervised physical exercise in patients with symptomatic coronary artery disease: reduction of stress-induced myocardial ischemia. Circulation 1988;77:172-81.

42. Niebauer J, Hambrecht R, Velich T, et al. Predictive value of lipid profile for salutary coronary angiographic changes in patients on a low-fat diet and physical exercise program. Am J Cardiol 1996;78:163-7.

43. Haskell WL, Alderman EL, Fair JM, et al. Effects of intensive multiple risk factor reduction on coronary atherosclerosis and clinical cardiac events in men and women with coronary artery disease. The Stanford Coronary Risk Intervention Project (SCRIP). Circulation 1994;89:975-90.

44. Hjermann I, Velve Byre K, Holme I, et al. Effect of diet and smoking intervention on the incidence of coronary heart disease. Report from the Oslo Study Group of a randomised trial in healthy men. Lancet 1981;2:1303-10.

45. Hooper L, Summerbell CD, Higgins JP, et al. Reduced or modified dietary fat for preventing cardiovascular disease. Cochrane Database Syst Rev 2001;3:CD002137.

46. Truswell AS. Review of dietary intervention studies: effect on coronary events and on total mortality. Aust N Z J Med 1994;24:98-106.

47. Mente A, de Koning L, Shannon HS, et al. A systematic review of the evidence supporting a causal link between dietary factors and coronary heart disease. Arch Intern Med 2009;169:659-69.

48. Schwingshackl L, Hoffmann G. Monounsaturated fatty acids and risk of cardiovascular disease: synopsis of the evidence available from systematic reviews and meta-analyses. Nutrients 2012:4:1989-2007.

49. Lichtenstein AH, Appel LJ, Brands M, et al. Diet and lifestyle recommendations revision 2006: a scientific statement from the American Heart Association Nutrition Committee. Circulation 2006;114:82-96.

50. Bantle JP, Wylie-Rosett J, Albright AL, et al. Nutrition recommendations and interventions for diabetes: a position statement of the American Diabetes Association. Diabetes Care 2008;31(Suppl 1):S61-78. 